\title{
Ernesto de Sola. Arquitecto
}

\author{
Sandra Gutiérrez Poizat \\ Universidad Centroamericana \\ "José Simeón Cañas", UCA
}

El 8 de noviembre de 2017, en el salón Ernesto Álvarez Córdova del Museo de Arte (MARTE) se presentó, a un reducido público, la monografía de la obra arquitectónica de Ernesto de Sola. La exquisita publicación ha sido producto del esfuerzo de diversos autores, entre arquitectos e historiadores, y contó con el patrocinio de la familia de Sola.

La reseña que a continuación se presenta hace una revisión tanto de los textos propuestos, como de las obras arquitectónicas seleccionadas en la publicación. En relación a los textos, me detengo en dos afirmaciones del prólogo que generalizan el impacto de la obra de Ernesto de Sola, sin tomar en cuenta otros factores que también inciden en dicho impacto. Destaco la invitación que hace el "Ensayo" a reflexionar sobre una arquitectura propia y apropiada al país. La "Introducción", la cual propone una clasificación bastante acertada de la obra, se intenta presentar de forma resumida sin descuidar los diversos niveles de análisis que plantea el autor. En cuanto a las obras arquitectónicas seleccionadas para ser presentadas en el libro material histórico de enorme calidad y muy bien conservado- propongo examinarlas a la luz de la propuesta clasificatoria de la introducción, lo cual no necesariamente se logra con fuerza en el libro. Finalmente, propongo al lector una valoración sobre el impacto de esta inédita publicación y la necesidad de hacer llegar este tipo de contenidos a públicos más amplios.

\section{Los textos: contextualización y análisis de la obra de Ernesto de Sola}

El "Prólogo", escrito por Luis creador de Ernesto de Sola y llega Salazar Retana, insiste en el carácter a afirmar que, a través de su obra, 
logra dar una "fisonomía moderna" a San Salvador, al mismo tiempo que propicia el "nacimiento de una nueva forma de vivir en el país" (Cañas Dinarte, Ferrufino, Salazar Retana y Regazzoli, 2016, p. 13). En el caso de la llamada "fisonomía moderna", la obra presentada, que comprende un período de 32 años, incide sin duda en ciertos cambios formales en la ciudad pero no la transforma en su totalidad. Kevin Lynch afirma que la ciudad se construye y mantiene por una diversidad de actores, cada uno con sus propios intereses y mecanismos de incidencia, algunos de ellos son públicos y otros privados, algunos dominantes y otros seguidores (Lynch, 1984, pp. 40-41). Los aportes de Ernesto de Sola, sin duda pioneros, deben verse en relación a la diversidad de propuestas provenientes de esos otros actores. Ernesto de Sola tiene la capacidad y los medios para producir una obra abundante desde la iniciativa privada, reforzando su figura de desarrollador o promotor urbano. Esta obra se localiza en espacios estratégicos privilegiados por la inversión privada, reforzando desde lo privado el discurso de progreso y modernidad promovido por el Estado. Sin embargo, más allá del impacto que su obra produce en partes de la ciudad, resulta arriesgado afirmar que solamente su obra le confiere una "fisonomía moderna" a San Salvador, habría que valorar esta obra en función, por ejemplo, de las inversiones públicas (sistema vial, espacios públicos, infraestructura deportiva y educativa, entre otros), que igualmente reforzaron el carácter moderno de la ciudad capital. En cuanto al planteamiento de propiciar el "nacimiento de una nueva forma de vivir en el país", las casas o villas, sin duda, introdujeron cambios en los estilos de vida de las familias que las habitaron. Estos cambios estaban, en alguna medida, en sintonía con las propuestas espaciales de la modernidad internacional, por ejemplo con la introducción de espacios para el vehículo o las nuevas propuestas de mobiliario y funcionalidad en las cocinas. Algunos de estos elementos se proponen, aunque en otra escala, en los conjuntos habitacionales desarrollados por el Estado en la primera mitad del siglo XX (contemporáneos a la obra de Sola). El momento histórico permite introducir en el país nuevos modelos de vivir en los espacios habitacionales, de Sola fue, según el espíritu de la época, uno de sus impulsores.

El "Ensayo", escrito por Carlos Cañas Dinarte, logra resaltar la figura de Ernesto de Sola como uno de los primeros salvadoreños profesionalizados en la carrera de arquitectura. Indica acertadamente que, previo a la existencia de la figura del arquitecto, las edificaciones eran confiadas a maestros de obra, constructores, albañiles e ingenieros. Por otra parte, contextualiza 
acertadamente la obra de Ernesto de Sola con respecto a esos otros actores que también hacen ciudad y que complementan y facilitan sus propuestas, como la construcción del Estadio Nacional "Flor Blanca", al cual se llegaba por medio de una "carretera moderna", o la actual alameda Franklin Delano Roosevelt. Ambas fueron inversiones públicas que motivaron a "un sector de la población y el gobierno" a "urbanizar y embellecer" la zona (Cañas Dinarte, Ferrufino, Salazar Retana y Regazzoli, 2016, p. 22). Finaliza su argumento invitando a una "reflexión necesaria y profunda" sobre la búsqueda de una arquitectura "propia y apropiada, precisa y eficiente" en relación a las condiciones naturales y de vida en El Salvador (Cañas Dinarte, Ferrufino, Salazar Retana y Regazzoli, 2016, p. 23).

La "Introducción"es una propuesta para analizar y reconocer los aportes de la obra de Ernesto de Sola. Nos invita a ver este legado como parte de la memoria de El Salvador, en particular la de San Salvador. Carlos Ferrufino se refiere a una especie de memoria latente, producto de la nostalgia por ciertas edificaciones del centro de San Salvador, o por la atracción que aún causan las casas de la colonia Flor Blanca, o por aquellos de los edificios diseñados por de Sola que aún siguen en uso. En este sentido, plantea que la publicación facilita, a través del análisis de su obra, reconocer sus aportes a la arquitectura salvadoreña del siglo XX. Para abordar dicho análisis propone cuatro aspectos: primero, una mirada general a los proyectos donde destacan algunas de sus características arquitectónicas principales, enfatiza la idea de recorrido histórico y subraya continuidades así como también rupturas. En segundo lugar, ofrece la contextualización de la obra en el marco de la arquitectura producida en el país entre 1930 y 1960. Como tercer punto, también examina la relación con las corrientes vigentes en la región durante el mismo período. Para finalizar, comparte una reflexión sobre los aportes a la arquitectura nacional y sus implicaciones para la práctica profesional en el futuro.

Para desarrollar estos cuatro aspectos, Ferrufino propone dos grandes aproximaciones: por un lado, las aproximaciones tipológicas, que desmembrarían la obra en residencias unifamiliares y edificios privados; por otra parte, la comprensión de los proyectos por medio del lenguaje arquitectónico, para lo cual se plantean tres orientaciones: una historicista o neocolonial, mayormente desarrollada en las residencias; otra anunciando una futura modernidad o art déco, presente en diversos edificios comerciales; y finalmente una moderna, en sintonía con la modernidad internacional. Estas orientaciones se detallan a continuación. 


\subsection{Casas Históricas}

Varios son los elementos a destacar de este recorrido histórico: los conjuntos habitacionales homogéneos en entornos urbanos; la transición de la casa-patio a la casa-villa, en la que el proyecto se coloca compactamente al centro del terreno, rodeado de jardines, en lugar de disponerse alrededor de uno o varios patios, y el uso de lenguajes arquitectónicos del pasado, neoclásicos o neocoloniales.

\subsection{Edificaciones protomodernas y Art déco}

En este caso se hace referencia primordialmente a sus edificios comerciales. En este nuevo recorrido histórico, el autor plantea un primer momento de transición entre los códigos históricos y la visión moderna de vanguardia, allí destacan varios elementos: el componente estructural, el tratamiento de las fachadas y núcleos de acceso, la relación edificio-calle, que mantiene la regularidad de la traza urbana; y, finalmente, el marcado gusto por la estética lineal.

\subsection{Edificios modernos}

Aquí se analizan proyectos realizados a partir de la segunda mitad del siglo XX. Se plantea al menos cinco novedades, con respecto a la obra previa: la ganancia en transparencia y liviandad de los edificios con la introducción de amplios ventanales de piso a viga; el uso de cortasoles que protegen los planos de vidrio y crean, a la vez, texturas y efectos de luz y sombra; la renuncia a cualquier elemento de decoración; la introducción de jardines tanto en fachadas como en el interior de las edificaciones, que retranquean al mismo tiempo el edificio con respecto a la calle; $y$, finalmente, la transición de una propuesta volumétrica regular y ortogonal a una más fluida, curvilínea. Todo esto se da en franca coordinación con las propuestas del International Style, y formando parte de una clara primera modernidad en El Salvador.

Ferrufino finaliza su "Introducción" planteando, por un lado, algunas características esenciales y, por otro, algunas carencias frecuentes, que como él mismo indica son "frecuentes en la arquitectura salvadoreña" (Cañas Dinarte, Ferrufino, Salazar Retana y Regazzoli, 2016, p. 34). Al mismo tiempo invita a reflexionar sobre temas válidos para el futuro de la arquitectura en El Salvador, entre los cuales destacan: el dominio de la escala doméstica en edificaciones de diferentes escalas, la relación con las condiciones locales a través del uso de elementos de protección, la valorización de las referencias históricas locales sin -por ellorenunciar al manejo de lenguajes internacionales, para terminar con 
saber apreciar la intensidad de la capacidad creativa de Ernesto de
Sola y la calidad del material gráfico mostrado en esta publicación.

\section{Las obras documentadas}

Como se resaltó al inicio de este resumen, el material grafico presentado es de especial valor en el esfuerzo de documentar y analizar la producción arquitectónica local. Tanto dibujos como planos han sido cuidadosamente preservados y transmiten con claridad y fuerza, el brío creador de Ernesto de Sola. Por otra parte, la colección es bastante completa y ello constituye un valioso material documental para seguir investigando.

Sin embargo, en cuanto a la publicación, considero que hay un desacierto al momento de presentar al lector esta formidable colección gráfica; el haber iniciado con los edificios y no con las residencias. Esta presentación invertida resta continuidad a la detallada propuesta de análisis planteada por Ferrufino, quien inicia, justamente, con las casas históricas; eso es lo que esperaría ver en primer lugar un lector aplicado que desea sumergirse en el análisis de la obra.

Esta consideración no resta valor a la calidad y diversidad del material en sí mismo. Seguramente, el lector se deleitará con la preciosa colección de fotos históricas, planos, bocetos, dibujos, que muestran además la rigurosidad del proceso creativo de Ernesto de Sola.

\section{Reflexión final}

Es evidente que estamos ante un formidable material histórico de enorme valor tanto para un público especializado que desea seguir investigando como para un público más amplio que desea conocer la historia de la arquitectura en $\mathrm{El}$ Salvador, sobre la cual hay muy poco publicado a la fecha. Es por ello que lamentamos que la obra no haya sido hasta ahora difundida y compartida nada más que con un reducido grupo. Esperaríamos, por tanto, que en un futuro próximo el libro se distribuya más ampliamente para verdaderamente cumplir con la importante función social y cultural que una obra de esta indiscutible calidad está llamada a desempeñar. 
Referencias bibliográficas

- Cañas Dinarte, C., Ferrufino, C., Salazar Retana, L. y Regazzoli, J. (2016). Ernesto de Sola. Arquitecto. San Salvador, El Salvador: Editorial Kalina.

- Lynch, K. (1984). Good City Form. Cambridge, Massachusetts, Estados Unidos: MIT Press. 